\title{
Simulação Analítica da Dispersão de Poluentes Considerando a Ascensão da Pluma
}

\author{
Renata Cezimbra ${ }^{1, *} \quad$ Daniela Buske $^{2} \quad$ Regis Quadros $^{2} \quad$ Fabrício Harter $^{3}$ \\ ${ }^{1}$ Graduanda em Engenharia Sanitária e Ambiental, UFPel \\ ${ }^{2}$ Depto de Matemática e Estatística, IFM, UFPel \\ ${ }^{3}$ Depto de Meteorologia, FMET, UFPel \\ 96010-610, Pelotas, RS \\ E-mail: renatacezimbra@hotmail.com,daniela.buske@ufpel.edu.br, \\ regis.quadros@ufpel.edu.br, fabricio.harter@ufpel.edu.br
}

\section{RESUMO}

Para muitas aplicações, como em emissões industriais, é necessário levar em conta o efeito do empuxo sobre a pluma de poluente. Quando a pluma de poluentes é mais quente que o ambiente (menos densa) ela tende a se elevar até uma camada onde se encontre em equilíbrio termodinâmico, a altura efetiva da fonte $\left(H_{e}\right)$ será a soma da altura real da fonte $\left(H_{s}\right)$ e o efeito de ascensão da pluma $(\Delta H)$. Dessa forma assumimos que, a certa distância da fonte, a pluma de material liberado em $H_{S}$ se comporta como uma pluma de mesma densidade que o ambiente, abandonada sem empuxo a uma altura $H_{e}$ [2]. Em casos de convecção forte $(h /|L|>10)$, a pluma terá uma ascensão final dada por

$$
\Delta H=4.3\left(\frac{F}{\bar{u} w_{*}}\right)^{3 / 5} h^{2 / 5}
$$

sendo $F$ um parâmetro de flutuabilidade definido como $F=g V_{i} r_{i}{ }^{2} \frac{\left(T_{i}-T_{a}\right)}{T_{i}} \quad$ onde $g$ é a aceleração da gravidade, $T_{i}, V_{i}, r_{i}$ e $T_{a}$ são a temperatura da fonte, a velocidade vertical de saída, raio da fonte e temperatura ambiente [3]. Para condições moderadamente convectivas a ascensão da pluma é dada como

$$
\Delta H=\left(\frac{F}{\bar{u} w_{d}^{2}}\right)^{\frac{3}{5}}\left(1+\frac{2 H_{s}}{\Delta H}\right)^{2}
$$

que pode ser resolvida interativamente, onde $w_{d}=0.4 w_{*}$ é a velocidade média dos downdrafts (correntes de ar descendentes). Para condições de estabilidade neutra a seguinte expressão para $\Delta H$ :

$$
\Delta H=1.3 \frac{F}{\bar{u} u_{*}^{2}}\left(1+\frac{H_{s}}{\Delta H}\right)^{2 / 3} .
$$

Weil [7] sugere que uma pluma tem a seguinte restrição para sua ascensão:

$$
\Delta H=0.62\left(h-H_{s}\right)
$$

Considerado o que foi exposto acima, Briggs [3] sugere que o valor final de $\Delta H$ deve ser o valor mínimo obtido com as equações (1)-(4). Esta sugestão é a mais prudente, pois na medida em que o efeito de ascensão é maior, menores serão os valores de concentração obtidos para níveis próximos do solo, diminuindo o risco de subestimar o valor destas concentrações.

Para analisar o efeito da ascensão da pluma em um modelo matemático, consideramos a equação de advecção-difusão bidimensional transiente,

$$
\frac{\partial C}{\partial t}+u \frac{\partial C}{\partial x}=\frac{\partial}{\partial z}\left(k_{Z} \frac{\partial C}{\partial Z}\right)
$$

onde $z$ é altura da camada limite atmosférica (CLA), $x$ é a direção do vento médio u, $K_{z}$ é o coeficiente de difusão turbulento dependente da altura z. A Eq. (5) esta sujeita a condição inicial nula; a condição de fonte $\bar{u} C(0, z, t)=Q \delta\left(z-H_{e}\right)$ em $x=0$, onde $Q$ é a taxa de emissão do poluente, $H e$ é a altura efetiva da fonte (onde é levado em conta o efeito do empuxo inicial);

\footnotetext{
* Bolsista de Iniciação Científica PROBIC/FAPERGS
} 
e as condições de contorno de fluxo nulo no solo e no topo da camada limite. A solução da equação de advecção-difusão (5) é conhecida na literatura e é obtida utilizando as técnicas da transformada de Laplace e GILTT (Generalized Integral Laplace Transform Technique) [6].

Como um exemplo de aplicação, apresentamos resultados preliminares de uma simulação de dispersão do $\mathrm{SO}_{2}$ emitido pela Usina Termelétrica Presidente Médici de Candiota/RS. Esta usina é uma importante fonte de liberação de $\mathrm{SO}_{2}$ na região. Foi um experimento de fonte alta, onde o $\mathrm{SO} 2$ foi liberado com empuxo de forma continua de uma chaminé com $150 \mathrm{~m}$ de altura a uma taxa de $0,7 \mathrm{~kg}^{-1}$. A velocidade de saída e a temperatura de exaustão são de aproximadamente $20 \mathrm{~m} . \mathrm{s}^{-1}$ e $420 \mathrm{~K}$, respectivamente.

Para podermos comparar os resultados obtidos pela GILTT com os dados da literatura assumimos que a concentração de poluentes possui uma distribuição gaussiana na direção y (solução 3D aproximada, conhecida como GILTTG). Nas simulações foram utilizados um perfil de vento potencia e coeficiente de difusão definido em Degrazia et. al. [4].

A tabela 1 mostra os resultados estatísticos [5] da comparação da solução com alguns valores do experimento de Candiota apresentados em Arbage et al. [1]. Observamos uma melhora significativa nos resultados em que o efeito da ascensão da pluma foi considerado (aumento da correlação (COR) e a diminuição do erro quadrático médio normalizado (NMSE)). As concentrações preditas apresentam valores maiores que as observadas. O modelo também apresentou fator de dois (FA2) de 60\%. Nosso próximo objetivo será o de ampliar esta aplicação, utilizando mais dados do sitio de Candiota.

Tabela 1: Dados estatísticos obtidos pelo modelo GILTTG.

\begin{tabular}{c|ccccc}
\hline & NMSE & COR & FA2 & FB & FS \\
\hline GILTTG sem ascensão da pluma & 5,36 & 0,57 & 0,4 & 1,3 & 1,6 \\
GILTTG com ascensão da pluma & 1,07 & 0,83 & 0,6 & 0,4 & 1,2 \\
\hline
\end{tabular}

Palavras-chave: Solução Analítica, Transformada de Laplace, Equação de Advecção-Difusão

\section{Referências}

[1] M.C. Arbage, G.A. Degrazia, O.L. Moraes, Simulação euleriana da dispersão local da pluma de poluente atmosférico de Candiota-RS. Rev. Bras. Meteorologia, vol. 21 (2), pp. 153-160, (2006).

[2] S. Pal. Arya, Air Pollution Meteorology and Dispersion. New York, USA: Oxford University Press, 310p., (1999).

[3] G.A. Briggs, Plume Rise Predictions, Lectures on Air Pollution and Environmental Impact Analyses, D.A. Haugen ed., Amer. Meteor. Soc., Boston, MA, pp. 59-111, (1975).

[4] G.A. Degrazia, H.F. Campos Velho, J.C. Carvalho, Nonlocal Exchange coefficients for the convective boundary-layer derived from spectral properties. C. Atmos. Physics, pp. 57-64, (1997).

[5] S.R. Hanna, Confidence limit for air quality models as estimated by bootstrap and jackknife resampling methods. Atmos. Environ., vol. 28, pp. 1385-1395, (1989).

[6] D.M. Moreira, M.T. Vilhena, D. Buske, T. Tirabassi, The state-of art of the GILTT method to simulate pollutant dispersion in the atmosphere. Atmos. Research, vol. 92, pp. 1-17, (2009).

[7] J.C. Weil, Assessmet of plume rise and dispersion models using LIDAR data, PPSP-MP-24. Prepared by Environmental Center, Martin Marietta Corporation, for Maryland Department of Natural Resources. (1979). 